\title{
Práticas Educativas Maternas e Comportamentos Infantis no Transtorno de Déficit de Atenção/Hiperatividade
}

\author{
Luciana Assis-Silva ${ }^{1}$ \\ Direção do Instituto Transformação, Salvador, Brasil \\ Curso de Psicologia da Faculdade Regional da Bahia - UNIRB, Salvador, Brasil \\ Patrícia Alvarenga \\ Departamento de Psicologia da Universidade Federal da Bahia, Salvador, Brasil
}

\begin{abstract}
Resumo
O estudo investigou diferenças nas práticas educativas de mães de crianças com Transtorno de Déficit de Atenção/Hiperatividade (TDAH) e mães de crianças sem TDAH. Participaram do estudo 19 díades mãe-criança divididas em dois grupos. Todas as díades foram formadas por meninos com idades que variaram entre seis e dez anos. O grupo clínico foi composto por nove díades com meninos que receberam o diagnóstico clínico de TDAH. O grupo não-clínico foi formado por dez díades com crianças sem o transtorno. As práticas educativas maternas e os comportamentos infantis foram avaliados em uma sessão de observação da interação mãe-criança. A observação foi composta por quatro episódios de interação: Brincadeira Livre, Guardar Brinquedos, Atividade de Cópia e Episódio de Cooperação. A sessão de observação foi filmada e teve duração aproximada de 40 minutos. Os comportamentos maternos foram codificados em nove categorias: controle ambíguo; controle coercitivo; insensibilidade; intrusividade; negatividade; permissividade; controle assertivo; envolvimento positivo e incentivo. Os comportamentos da criança foram avaliados através de nove categorias: desobediência passiva; inadequação; insensibilidade; negativismo; assertividade; envolvimento positivo; obediência; solicitação e brincadeira independente. Os resultados obtidos revelaram apenas uma diferença significativa, que indicou que as mães do grupo clínico apresentaram maior frequência de controle assertivo do que as mães do grupo não-clínico. Discute-se o possível caráter aversivo do uso do controle assertivo em excesso e seu impacto sobre o comportamento infantil e a relevância de um diagnóstico criterioso para o avanço dos estudos nesta area.
\end{abstract}

Palavras-chave: Transtorno de Déficit de Atenção/Hiperatividade, práticas educativas maternas, comportamento infantil, desenvolvimento infantil.

\section{Childrearing Practice and Child Behavior on Attention Deficit Hyperactivity Disorder}

\begin{abstract}
The present study investigated differences in the use of childrearing practices by mothers whose children have been diagnosed with Attention Deficit Hyperactivity Disorder (ADHD) and by mothers whose children have not been diagnosed with ADHD. Participants in the study were 19 mother-child dyads divided into two groups. All dyads included boys between the ages of six and ten. The clinical group

Endereço para correspondência: Rua Clínio de Jesus, 618, Apto. 101, Salvador, BA, Brasil 40301200. E-mail: lumaria_ssa@yahoo.com.bre palvarenga66@gmail.com

Este estudo foi baseado na Dissertação de Mestrado da primeira autora, realizada sob a supervisão da segunda autora, apresentada no Programa de Pós-Graduação em Psicologia da Universidade Federal da Bahia, Salvador, BA.
\end{abstract}


was composed of nine dyads with children diagnosed with ADHD. The non-clinical group included ten dyads with children without the disorder. An observation session of mother-child interaction was carried out in order to analyze maternal childrearing practices and child behaviors. The observation consisted of four interaction episodes: free play, putting away toys, copying activity, and cooperation. The interaction was filmed and lasted approximately 40 minutes. Maternal behaviors were coded into nine categories: ambiguous control; coercive control; insensitivity; intrusiveness; negativity; permissiveness; assertive control; positive involvement; and encouragement. Child behaviors were assessed using nine categories: noncompliance; inadequacy; insensitivity; negativism; assertiveness; positive involvement; compliance; request; independent play. The results revealed only one difference between the two groups: mothers from the clinical group displayed a higher frequency of assertive control than did mothers from the non-clinical group. The relevance of a rigorous diagnostic process for the advancement of research in this field is discussed.

Keywords: Attention Deficit/Hyperactivity Disorder, maternal childrearing practices, child behavior, child development.

\section{Prácticas Educativas de Madres y los Comportamientos Infantiles en Trastorno por Déficit de Atención/Hiperactividad}

\section{Resumen}

El estudio investigó las diferencias en las prácticas educativas de las madres de niños con Trastorno por Déficit de Atención/Hiperactividad (TDAH) y madres de niños sin TDAH. Los participantes del estudio fueron 19 díadas madre-hijo divididas en dos grupos. Todas las díadas fueron formadas por niños de edades comprendidas entre los seis y los diez años. El grupo clínico se compuso de nueve díadas con niños que recibieron un diagnóstico clínico de TDAH. El grupo no clínico fue formado por diez diadas con niños sin el trastorno. Las prácticas educativas maternas y los comportamientos infantiles fueron evaluadas en una sesión de observación de la interacción madre-hijo. La observación consistió en cuatro episodios de interacción: Juego Libre, Guardar Juguetes, Actividad de Copia, Episodio de Cooperación. La sesión fue grabada en vídeo y la observación duró aproximadamente 40 minutos. Las conductas maternas fueron codificadas en nueve categorías: control ambiguo; control coercitivo; insensibilidad; intrusión; negatividad; permisividad; control assertivo; participación positiva e incentivo. Os comportamentos da criança foram avaliados através de nove categorias: desobediência passiva; inadequação; insensibilidade; negativismo; assertividade; envolvimento positivo; obediência; solicitação e brincadeira independente. Los resultados revelaron sólo una diferencia significativa, que indicó que las madres en el grupo clínico tuvieron una mayor frecuencia de control asertivo que las madres de grupo no clínico. Se discute la importancia de un diagnóstico riguroso para el avance de los estudios en esta área.

Palabras claves: Trastorno por Déficit de Atención/Hiperactividad, prácticas educativas maternas, comportamiento infantil, desarrollo infantil.

O Transtorno de Déficit de Atenção/Hiperatividade (TDAH) é um quadro sindrômico, composto de sinais e sintomas comportamentais relacionados à desatenção, à hiperatividade e à impulsividade (Cypel, 2001; Rohde, Barbosa, Tramontina, \& Polanczyk, 2000). O DSM-IV-TR (American Psychiatric Association [APA], 2000) apresenta três tipos distintos de TDAH, de acordo com a predominância de sintomas: tipo predominantemente desatento, tipo predominantemente hiperativo-impulsivo e tipo combinado ou misto. Existem ainda outras especificações e categorias aplicáveis a casos que não satisfazem todos os critérios diagnósticos. Os sintomas do transtorno costumam iniciar normalmente antes dos sete anos de idade, embora a maioria dos 
pacientes seja diagnosticada apenas depois de alguns anos. Esses sintomas podem ser observados em situações distintas, como por exemplo, na escola ou no ambiente doméstico (Poeta \& Rosa, 2004).

A prevalência do TDAH varia de 1 a $20 \%$ em diferentes países (Barkley, 2008; Golfeto \& Barbosa, 2003). A grande amplitude dessa variação pode ser explicada em parte por variáveis culturais, e também pelos diferentes critérios e métodos utilizados para definir e identificar o TDAH, além do grau de concordância exigido entre as avaliações dos pais, professores e profissionais com relação à sintomatologia da criança (Lambert, Sandoval, \& Sassone, 1978). A proporção de meninos e meninas afetada varia de aproximadamente 2:1 até 9:1 conforme o tipo de transtorno (predominantemente desatento, predominantemente hiperativo-impulsivo ou combinado) e também de acordo com a natureza da amostra (comunitária, clínica ou escolar). Contudo, é possível que as meninas sejam subdiagnosticadas por apresentarem com maior freqüência o TDAH com predomínio desatento, e por terem poucos sintomas de agressividade/ impulsividade e do Transtorno de Conduta, um quadro frequentemente associado ao TDAH em meninos. Por essas reazões os sintomas das meninas teriam menor impacto em seu ambiente social e elas seriam encaminhadas para tratamento com menor freqüência (Rohde \& Halpern, 2004). Independentemente do sexo, o TDAH está relacionado a vários prejuízos para o desenvolvimento infantil como perturbações motoras, dificuldades de aprendizagem e fracasso escolar (Artigas-Pallarés, 2003; Sell-Salazar, 2003).

O diagnóstico do TDAH é fundamentalmente clínico, baseando-se em critérios operacionais claros e bem definidos, provenientes de sistemas classificatórios como o DSM-IV-TR (APA, 2000). Esse sistema propõe a necessidade de pelo menos seis sintomas de desatenção e/ou seis sintomas de hiperatividade/impulsividade para o diagnóstico. Além disso, alguns sintomas de hiperatividade-impulsividade ou desatenção, causadores do comprometimento, devem estar presentes antes dos sete anos. Algum comprometimento causado pelo transtorno deve estar presente em dois ou mais contextos, e deve ha- ver claras evidências de comprometimento clinicamente importante no funcionamento social, acadêmico ou ocupacional (APA, 2000).

Os determinantes do TDAH ainda não são conhecidos, apesar do grande número de estudos já realizados (Barkley, 2008; Roman, Schmitz, Polanczyk, \& Hutz, 2003; Tannock, 1998). Entretanto, a influência de fatores genéticos, neurológicos e ambientais no seu desenvolvimento é amplamente aceita na literatura (Barkley, 2002, 2008; Tannock, 1998). Entre as variáveis ambientais, as relações familiares, a interação pais criança e, mais especificamente, as práticas educativas parentais, parecem desempenhar um papel importante.

As práticas educativas parentais são estratégias que os pais utilizam com o objetivo de promover a socialização dos filhos (Alvarenga \& Piccinini, 2001; Kunrath, Wagner, \& Jou, 2006; Pacheco, Silveira, \& Schneider, 2008). Os pais procuram direcionar os comportamentos dos filhos com o objetivo de fazer com que eles sigam certos princípios morais e adquiram um repertório comportamental que lhes possibilitem autonomia e responsabilidade para o bom convívio social. Além disso, os pais tentam suprimir ou reduzir comportamentos inadequados dos filhos (Alvarenga \& Piccinini, 2001). Alvarenga e Piccinini (2007) desenvolveram, a partir das perspectivas teóricas de Hoffman (1979) e de Patterson, Reid e Dishion (1992), uma classificação que contemplasse de forma mais ampla as práticas educativas parentais relacionadas aos problemas de comportamento distinguindo-as daquelas relacionadas ao desenvolvimento da competência social. Esses autores utilizaram os seguintes critérios na construção dessa classificação: a presença ou ausência da coerção, o fato de as práticas serem ou não contingentes, a existência ou não de intrusividade (tendência ao controle exagerado dos comportamentos da criança), a autorização da autonomia, o tipo de envolvimento afetivo (positivo ou negativo) e o caráter assertivo das práticas (clareza e firmeza no uso de comandos e instruções). O termo práticas educativas facilitadoras do desenvolvimento social é utilizado para designar estratégias como a assertividade, a sensibilidade, o envolvimento positivo e as orientações e explicações utilizadas 
na regulação do comportamento da criança. Já o termo práticas educativas não facilitadoras do desenvolvimento social é utilizado para designar as estratégias coercitivas, intrusivas, ambíguas ou permissivas de controle (Alvarenga \& Piccinini, 2007, 2009).

$\mathrm{Na}$ literatura que examina as relações entre variáveis ligadas ao comportamento parental e o TDAH, é possível identificar duas tendências distintas. A primeira delas pressupõe que o comportamento parental contribua para acentuar a apresentação e continuidade dos sintomas do transtorno (Carlson, Jacobvitz, \& Sroufe, 1995; Patterson, DeGarmo, \& Knutson, 2000). A segunda tendência compreende que o comportamento parental estaria associado apenas ao surgimento de transtornos comumente associados ao TDAH, como o Transtorno Desafiador Opositivo (TDO) e o Transtorno da Conduta (TC; Barkley, 2002; Whalen \& Henker, 1999).

Adeptos da primeira tendência mencionada acima, Carlson et al. (1995), afirmam que as disfunções nas relações familiares, bem como as práticas parentais, podem servir como fatores de risco que interagem com as predisposições genéticas para exacerbar a apresentação e a continuidade dos sintomas do TDAH. De acordo com esse modelo, os fatores familiares ampliariam e manteriam influências sobre o transtorno durante o curso do desenvolvimento. Em algumas circunstâncias, a criança poderia ter relativamente baixa predisposição ao transtorno, mas um ambiente familiar caótico e pais com poucas habilidades de regular a conduta infantil serviriam para exacerbar a desatenção, a impulsividade e a hiperatividade para um nível clinicamente relevante.

Nessa mesma perspectiva, Patterson et al. (2000) levantam a hipótese de que a relação entre o TDAH e transtornos como o TDO e o TC, que se caracterizam pela presença de comportamentos antissociais, não se trata de comorbidade, já que este termo implica diferenças causais nos mecanismos responsáveis pelo surgimento e manutenção dos transtornos. Para os autores, a hiperatividade representa um estágio inicial e o comportamento antissocial um estágio mais avançado de um mesmo processo. A hipótese dos autores é a de que esse processo deve começar cedo, durante os anos pré-escolares, mais ou menos aos dez meses de idade, quando uma criança com alto nível de atividade física e emocionalidade negativa, entre outras possíveis características de temperamento difícil, está interagindo com cuidadores pouco responsivos. Esse processo evoluiria rapidamente, e mais ou menos aos 24 meses de idade, a criança em risco poderia tornar-se tanto coercitiva (com comportamentos aversivos como desobediência, birra, etc.), quanto socialmente inábil (dificuldades na linguagem, atenção pobre), o que caracteriza tanto crianças antissociais quanto crianças hiperativas. Esses comportamentos, quando submetidos a um ambiente caracterizado por práticas educativas não contingentes e ineficazes e pouco envolvimento parental ocasionariam o surgimento de um quadro típico de conduta antissocial. Porém, estima-se que apenas cerca de metade das crianças que têm hiperatividade irá desenvolver comportamento antissocial.

No entanto, outros pesquisadores acreditam que a parentalidade não faz parte do conjunto de preditores do TDAH (Barkley, 2002; Johnston \& Jassy, 2007; Loeber, Green, Lahey, Frick, \& Mcburnett, 2000; Whalen \& Henker, 1999). Para eles, as dificuldades de interação entre pais e criança estão associadas à presença da comorbidade do TDO ou do TC em crianças com TDAH (Whalen \& Henker, 1999) e não ao surgimento de sintomas de desatenção, hiperatividade e impulsividade. De acordo com esse modelo, fatores de natureza genética interagem com fatores familiares e práticas parentais para criar, ou impedir, o desenvolvimento de problemas de conduta (Loeber et al., 2000). De acordo com Johnston e Jassy (2007), as características do TDAH aparecem cedo na vida da criança e se constituem em desafios para os pais, que têm dificuldades em manter práticas educativas consistentes e responsivas. Por sua vez, a presença de práticas educativas severas ou inconsistentes contribui para o desenvolvimento do comportamento opositor e de problemas de conduta em geral na criança.

De acordo com Johnston e Mash (2001), o TDAH pode representar um risco para o desenvolvimento de problemas de conduta, pelo menos, através de dois mecanismos, que pro- 
vavelmente, funcionam simultaneamente: (a) a presença da desatenção e da impulsividade torna a criança mais suscetível a apresentação de práticas parentais que estão associadas a problemas de conduta (ex. disciplina inconsistente e monitoramento pobre dos comportamentos da criança), e (b) o TDAH da criança pode atuar como um forte estressor que desencadeia uma crise na parentalidade prejudicando as práticas educativas e habilidades dos pais para regular a conduta da criança (ex. disciplina dura ou reativa).

Não existe um consenso na literatura a respeito de como as práticas educativas influenciam o TDAH, nem achados conclusivos que possam apoiar de forma consistente uma dessas duas hipóteses. Diferentes estudos apontam para diferentes vertentes a respeito de como as práticas parentais podem estar associadas ao desenvolvimento do transtorno e ao aparecimento dos problemas de conduta, sendo necessário um maior aprofundamento nos estudos nessa área (Johnston \& Mash, 2001). Neste estudo adota-se a hipótese de que as práticas educativas parentais estão envolvidas na manutenção do TDAH, bem como poderiam agravar seus sintomas. Mesmo que práticas mais severas, menos eficazes e pouco contingentes possam ser evocadas pelos sintomas apresentados pelas crianças, pressupõe-se que a inabilidade parental para empregar práticas mais eficazes na regulação da conduta infantil, contribua para manter e exacerbar a sintomatologia.

Em muitas pesquisas, foram obtidos achados que sugerem que mães de crianças com TDAH são mais intrusivas, exigentes, negativas e aversivas. Além disso, essas mães fazem menos aprovações e incentivos e expressam menos afeto positivo em comparação com grupos de mães de crianças sem TDAH (Alizadeh, Applequist, \& Coolidge, 2007; DuPaul, McGoey, Eckert, \& VanBrakle, 2001; Gau, 2007; Keown \& Woodward, 2002; Mash \& Johnston, 1982). Crianças com TDAH, por sua vez, são mais negativas e nervosas, exigem mais ajuda e atenção, e são menos complacentes quanto às instruções e pedidos da mãe em comparação com crianças que não apresentam o transtorno (DuPaul et al., 2001; Keown \& Woodward, 2002; Kunrath et al., 2006; Mash \& Johnston, 1982).
Nesse contexto, é importante considerar as práticas educativas que os pais utilizam para promover o desenvolvimento social pleno de seus filhos, com a finalidade de prevenir dificuldades de relacionamento tanto na família, quanto em outros ambientes (Kunrath et al., 2006). Além disso, é importante identificar as práticas educativas mais utilizadas pelos pais de crianças com TDAH para propor programas de treinamento para pais, que visem à promoção de práticas educativas que possibilitem o melhor desenvolvimento da criança. Soma-se a isso o fato de que existem poucos estudos no Brasil referentes às práticas educativas de pais de crianças com TDAH (Kunrath et al., 2006).

Este estudo teve como objetivo investigar diferenças nas práticas educativas de mães de meninos com TDAH e mães de meninos sem TDAH. A hipótese foi de que as mães de meninos com TDAH seriam mais intrusivas, mais negativas e utilizariam mais o controle coercitivo. Além disso, as mães expressariam menos envolvimento positivo, em comparação com o grupo de mães de crianças sem TDAH. Em relação ao comportamento das crianças com TDAH, a hipótese inicial do estudo foi de que portadores do transtorno apresentariam mais comportamentos negativistas e inadequados e mais desobediência passiva do que as crianças sem TDAH.

\section{Método}

\section{Participantes}

Participaram do estudo 19 díades mãe-criança, com crianças do sexo masculino de seis a dez anos, divididas em dois grupos. O grupo clínico foi composto por nove díades com crianças que receberam diagnóstico clínico de TDAH. O grupo não-clínico foi formado por dez díades com crianças sem o transtorno.

Oito dos nove meninos que participaram do estudo receberam diagnóstico de TDAH do tipo misto/combinado e apenas um recebeu diagnóstico de TDAH do tipo hiperativo. Além disso, em relação à comorbidade, dois deles apresentaram também sintomas de TDO. As análises estatísticas (boxplot) revelaram que as duas díades com crianças que tinham sintomas de TDO não apresentavam valores extremos em nenhum dos 
comportamentos infantis e práticas educativas maternas avaliados. Por esta razão, ambos os casos foram mantidos na amostra.

A Tabela 1 apresenta os dados demográficos dos participantes do estudo. No grupo clínico, a idade das crianças variou entre seis e dez anos $(M=8,55, D P=1,42)$ e no grupo não-clínico, a variação foi entre sete e dez anos $(M=8,5, D P$ $=1,26)$. Seis $(66,7 \%)$ meninos do grupo clínico estudavam em escolas particulares, enquanto to- das as crianças do grupo não-clínico estudavam em escolas públicas. No grupo clínico, a idade da mãe variou entre 24 e 42 anos $(M=34,44$, $D P=7,28)$, já no grupo não-clínico, a idade materna variou entre 28 e 49 anos $(M=38,80$, $D P=7,16)$. Em relação à escolaridade da mãe, a média de anos de estudo foi de nove anos $(D P$ $=3,27)$ no grupo clínico, e de 7,7 anos $(D P=$ $2,67)$ no grupo não-clínico. Nos grupos clínico e não-clínico, seis mães trabalhavam fora de casa $(66,7 \%$ e $70 \%$ respectivamente).

Tabela 1

\section{Características Demográficas dos Participantes}

\begin{tabular}{|c|c|c|c|c|}
\hline & Grupo Clínico (n=9) & Grupo Não-Clínico $(n=10)$ & & \\
\hline \multicolumn{5}{|l|}{ Idade da criança } \\
\hline$M(D P)$ & $8,55(1,42)$ & $8,50(1,27)$ & $U=43$ & $p=0,90$ \\
\hline$M d$ & 9 & 8,5 & & \\
\hline \multicolumn{5}{|l|}{ Tipo de escola } \\
\hline Particular & $6(66,7 \%)$ & $0(0 \%)$ & $X^{2}=9,74^{*}$ & $p=0,003$ \\
\hline Pública & $3(33,3 \%)$ & $10(100 \%)$ & & \\
\hline \multicolumn{5}{|c|}{ Escolaridade da criança } \\
\hline$M(D P)$ & $2(1,32)$ & $2,6(0,97)$ & $U=27,5$ & $p=0,16$ \\
\hline$M d$ & 2 & 2,5 & & \\
\hline \multicolumn{5}{|l|}{ Idade da mãe } \\
\hline$M(D P)$ & $34,44(7,28)$ & $38,80(7,16)$ & $U=29$ & $p=0,21$ \\
\hline$M d$ & 33 & 37 & & \\
\hline \multicolumn{5}{|c|}{ Escolaridade da mãe em anos } \\
\hline$M(D P)$ & $9(3,27)$ & $7,70(2,67)$ & $U=29,5$ & $p=0,21$ \\
\hline Md & 11 & 8 & & \\
\hline \multicolumn{5}{|l|}{ Ocupação da mãe } \\
\hline Não trabalha fora & $3(33,3 \%)$ & $3(30 \%)$ & $X^{2}=0,24^{*}$ & $p=1,00$ \\
\hline Trabalha fora & $6(66,7 \%)$ & $7(70 \%)$ & & \\
\hline \multicolumn{5}{|l|}{ Situação conjugal } \\
\hline Solteira & $5(55,6 \%)$ & $1(10 \%)$ & $* *$ & \\
\hline Morando com o pai & $3(33,3 \%)$ & $9(90 \%)$ & & \\
\hline $\begin{array}{l}\text { Morando com outro } \\
\text { homem }\end{array}$ & $1(11,1 \%)$ & $0(0 \%)$ & & \\
\hline \multicolumn{5}{|l|}{ Idade do pai } \\
\hline$M(D P)$ & $37,33(8,06)$ & $40,60(8,77)$ & $U=35,5$ & $p=0,45$ \\
\hline$M d$ & 37 & 40 & & \\
\hline
\end{tabular}


Escolaridade do pai em anos

$M(D P)$

$M d$

Ocupação do pai

Não trabalha fora

Trabalha fora

Número de irmãos

$M(D P)$

$M d$

Renda familiar em reais

$M(D P)$

$M d$
$8,11(3,10)$

8

$2(22,2 \%)$

$7(77,8 \%)$

$1,11(1,61)$

1

$838,88(394,66)$

850
$8,90(2,02)$

$U=39,5$

$p=0,66$

8

$$
\begin{gathered}
0(0 \%) \\
10(100 \%)
\end{gathered}
$$

$X^{2}=2,48^{*}$

$p=0,21$

$3,30(2,16)$

$U=11,5 \quad p=0,004$

2,5

$867,00(407,27)$

$U=44,5 \quad p=0,968$

810

Notas. ${ }^{*} g l=1 .{ }^{*}$ O teste qui-quadrado não pôde ser utilizado devido à baixa frequência esperada em algumas células.

A Tabela 2 apresenta dados sobre a existência de transtornos mentais e problemas com álcool ou droga nas famílias em ambos os grupos. Enquanto, no grupo clínico, sete mães $(77,8 \%)$ relataram ter algum parente com queixas de transtornos mentais, no grupo não-clínico, apenas duas mães $(20 \%)$ relataram o mesmo. Em relação à existência de pessoas com problemas com álcool ou drogas na família, no grupo clínico, sete mães $(77,8 \%)$ apontaram o problema, enquanto no grupo não-clínico, apenas três mães (30\%) fizeram esse tipo de relato.

A análise com o teste de Mann-Whitney e com o teste qui-quadrado das diferenças nos da- dos demográficos e problemas na família revelou algumas diferenças entre os grupos clínico e não-clínico. Na variável tipo de escola, foi constatada uma diferença significativa $\left(X^{2}=9,74, p\right.$ $=0,004)$, que indica um número maior de meninos estudando em escolas particulares no grupo clínico. Na variável número de irmãos, houve diferença significativa $(U=11,5, p=0,003)$, indicando um maior número de irmãos no grupo não-clínico. Outra diferença significativa foi encontrada na variável transtornos mentais na família $\left(X^{2}=4,33, p=0,02\right)$, indicando que as famílias de crianças com TDAH apresentavam um número maior de parentes com esse tipo de queixa.

Tabela 2

Queixas de Transtornos Mentais e Problemas com Álcool ou Drogas na Família

$$
\text { Grupo Clínico }(n=9) \quad \text { Grupo Não-Clínico }(n=10)
$$

\begin{tabular}{lllll}
\hline Transtornos mentais & & & & \\
Sim & $7(77,8 \%)$ & $2(20 \%)$ & $X^{2}=6,34$ & $p=0,02$ \\
Não & $2(22,2 \%)$ & $8(80 \%)$ & & \\
Álcool ou drogas & & & & \\
Sim & $7(77,8 \%)$ & $3(30 \%)$ & $X^{2}=4,33$ & $p=0,07$ \\
Não & $2(22,2 \%)$ & $7(70 \%)$ & & \\
\hline
\end{tabular}

Nota. $g l=1$. 


\section{Delineamento e Procedimento}

As díades do grupo clínico foram selecionadas no Ambulatório de Transtorno de Déficit de Atenção e Hiperatividade vinculado ao Serviço de Psiquiatria do Hospital Universitário Edgar Santos (Salvador/BA/BR) que atende a população de baixa renda. Todas as crianças que compuseram o grupo clínico passaram por um criterioso diagnóstico no referido ambulatório, composto por várias etapas. Na triagem inicial, realizava-se uma entrevista padrão sobre o desenvolvimento neuropsicomotor, histórico escolar, histórico da queixa e o preenchimento do Questionário Swanson, Nolan e Pelham (SNAP IV). Na etapa seguinte, o paciente passava por uma triagem neuropsicológica para estimativa de QI. Havendo indícios de TDAH, o paciente era encaminhado para uma triagem neuropsicológica mais abrangente, que incluía avaliação da atenção e da concentração, entre outros aspectos, além de triagem fonoaudiológica e psicopedagógica, e uma entrevista diagnóstica, o Schedule for Affective Disorders and Schizophrenia for School-Age Children/ Present and Lifetime Version (K-SADS-PL). Depois disso, o paciente era encaminhado para o psiquiatra, que solicitava avaliação oftalmológica e audiométrica, antes de concluir o diagnóstico. Após o diagnóstico clínico, as mães das crianças eram encaminhadas para a responsável pelo presente projeto de pesquisa e respondiam novamente o questionário SNAP IV, no qual o escore obtido deveria ser superior a 1,78 para sintomas de desatenção e superior a 1,44 para sintomas de hiperatividade e impulsividade. A média obtida pelo grupo clínico no questionário SNAP IV para sintomas de desatenção foi de 2,49 (DP=0,39) e de 2,61 ( $D P$ $=0,42)$ para sintomas de hiperatividade. Além disso, a criança não deveria estar tomando medicação estimulante. Nenhuma das crianças do grupo clínico tomava medicação psicotrópica no momento da coleta de dados.

As díades do grupo não-clínico foram selecionadas em uma escola pública do município de Salvador que atende a população de baixa renda. Inicialmente, crianças do sexo masculino, que não possuíam queixas de déficit de atenção e hiperatividade, foram indicadas pelas professoras. Além disso, a criança deveria obter escore no questionário SNAP IV inferior a 1,78 para sintomas de desatenção e escore inferior a 1,44 para sintomas de hiperatividade e impulsividade. A média do grupo não-clínico no questionário SNAP IV para sintomas de desatenção foi de $0,64(D P=0,29)$ e de $0,68(D P=0,29)$ para sintomas de hiperatividade. Nenhuma das crianças do grupo não-clínico tomava medicação psicotrópica no momento da coleta de dados.

As díades foram selecionadas e contatadas a partir de informações obtidas através da escola e do ambulatório. É importante ressaltar que, na escola, a pesquisadora realizou uma palestra para os professores descrevendo os principais sintomas e características do TDAH. Após a palestra, a pesquisadora pediu aos professores que, de acordo com seu julgamento, indicassem crianças que não apresentassem problemas de comportamento, tais como: defícit de atenção, hiperatividade, agressividade, irritabilidade, entre outros. A composição do grupo não-clínico foi feita a partir dessa indicação feita pelos professores.

O primeiro contato com as mães de ambos os grupos aconteceu por telefone, quando foram convidadas a participar do estudo. Para aquelas que concordaram, foi agendada uma visita domiciliar. Na data e horário agendados, a pesquisadora foi à casa da díade. As mães assinaram o Termo de Consentimento Livre e Esclarecido, enquanto as crianças manifestaram verbalmente sua concordância em participar da pesquisa. Após assinar o termo, a mãe respondeu a Ficha de Informações Demográficas da Família e o Questionário $S N A P-I V$. Após a aplicação do questionário, foi realizada a Observação da Interação Mãe-criança para análise das práticas educativas maternas e dos comportamentos infantis, dividida em quatro etapas: brincadeira livre, guardar brinquedos, atividade de cópia e episódio de cooperação. As observações foram filmadas.

O presente estudo foi aprovado pelo Comitê de Ética da Faculdade de Filosofia e Ciências Humanas/Instituto de Psicologia da Universidade Federal da Bahia em 03 de Maio de 2010. 


\section{Instrumentos}

Ficha de Informações Demográficas da Família. Visou obter informações demográficas da família, tais como: idade, profissão e nível de instrução dos pais, renda familiar, sexo e idade da criança.

Questionário Swanson, Nolan e Pelham (SNAP-IV; Swanson, 1992). É um questionário construído a partir dos sintomas do transtorno de déficit de atenção/hiperatividade descritos no DSM-IV-TR (APA, 2000). No presente estudo, foi utilizada a tradução validada pelo GEDA Grupo de Estudos do Déficit de Atenção da Universidade Federal do Rio de Janeiro (UFRJ) e pelo Serviço de Psiquiatria da Infância e Adolescência da Universidade Federal do Rio Grande do Sul (UFRGS; Mattos, Serra-Pinheiro, Rohde, $\&$ Pinto, 2006). O questionário tem 18 questões relativas a sintomas de desatenção, hiperatividade e impulsividade. Para cada questão são oferecidas quatro alternativas de resposta: "nem um pouco", "só um pouco", "bastante" ou "demais". As questões de um a nove são relativas aos sintomas de desatenção e as questões de dez a 18 são referentes a sintomas de hiperatividade e impulsividade. O escore em cada sub-escala é calculado somando-se os pontos em cada item e dividindo-se este valor pelo número total de questões. Se o escore total da criança na sub-escala de desatenção for maior que 1,78 existem mais sintomas de desatenção do que o esperado numa criança ou adolescente. Se o escore total da criança na sub-escala de hiperatividade e impulsividade for maior que 1,44 existem mais sintomas de hiperatividade/impulsividade do que o esperado numa criança ou adolescente (http:// www.adhd.net/snap-iv-instructions.pdf).

Observação da Interação Mãe-Criança. Foi realizada uma sessão de observação da interação da díade com objetivo de analisar as práticas educativas maternas e os comportamentos da criança. $\mathrm{O}$ roteiro da observação foi elaborado com base nos estudos de Keown e Woodward (2002), Landau, Amiel-Laviad, Berger, AtzabaPoria e Auerbach (2009) e Melnick e Hinshaw (2000). A observação foi composta por quatro episódios de interação: Brincadeira Livre, Guardar Brinquedos, Atividade de Cópia e Episódio de Cooperação. Esses quatro contextos foram escolhidos por proporcionarem variados tipos de interação mãe-criança de acordo com os estudos citados anteriormente. A sessão de observação foi filmada e teve duração aproximada de $40 \mathrm{mi}$ nutos. A seguir serão descritos os quatro episódios que constituíram a sessão de observação.

1. Brincadeira livre: As mães receberam a instrução de brincar com a criança livremente, utilizando alguns brinquedos que foram fornecidos pela pesquisadora (jogo com peças coloridas de encaixe, dois bonecos, dois carrinhos, uma bola, hidrocor e papel). O episódio de brincadeira livre teve duração de dez minutos.

2. Guardar brinquedos: Após a finalização dos dez minutos de brincadeira livre, a mãe deveria pedir à criança para guardar os brinquedos numa sacola.

3. Atividade de cópia: A criança recebia um lápis, uma borracha, um apontador e uma folha de papel com a impressão de sete figuras: triângulo, quadrado, círculo, hexágono, octógono, coração e cruz. A mãe deveria orientar a criança a fazer quatro cópias de cada uma das figuras na própria folha em que estavam impressas (Apêndice).

4. Episódio de cooperação. Nesta etapa da observação, mãe e criança deveriam montar juntas um quebra-cabeça de 30 peças.

\section{Procedimento de Análise de Dados}

A análise das práticas educativas e comportamentos infantis foi realizada de acordo com os critérios de análise por intervalos (Bakeman \& Gottman, 1999). Foram analisados seis minutos em cada episódio (três minutos iniciais e três finais). A análise foi feita em intervalos de 30 segundos durante os quais foram codificadas as práticas educativas da mãe e os comportamentos da criança. A estrutura de categorias utilizadas para análise foi elaborada com base nos estudos de diversos autores (Alvarenga \& Piccinini, 2009; Keown \& Woodward, 2002; Landau et al., 2009). Os comportamentos maternos foram codificados em nove categorias: controle ambíguo (formas indiretas e ambíguas de regular o comportamento da criança, como ordens, 
pedidos, proibições e sugestões vagas, indiretas ou confusas e comandos em forma de pergunta); controle coercitivo (tentativas de regular o comportamento da criança com estimulação aversiva); insensibilidade (logo após uma resposta de assertividade ou de envolvimento positivo da criança, a mãe persiste no comportamento anterior ou ignora o comportamento do filho); intrusividade (comportamento da mãe que indica que ela não percebe ou simplesmente não responde à necessidade de autonomia da criança ou a seus desejos e pontos de vista, impondo a sua própria vontade ou emitindo a resposta pela criança); negatividade (declarações verbais ou comportamentos não verbais que indiquem desânimo, não aceitação, desaprovação, comentários depreciativos ou irônicos em relação à criança ou ao seu comportamento); permissividade (comportamentos maternos que reforçam comportamentos inadequados ou negativos da criança); controle assertivo (comandos, proibições diretas, claras e precisas, sem uso de controle aversivo); envolvimento positivo (manifestações de afeto positivo por meio de comportamentos não-verbais e verbais); incentivo (declarações verbais ou não verbais para indicar encorajamento, aceitação ou aprovação dos comportamentos da criança). A categoria sem resposta foi utilizada quando a mãe não apresentava nenhum tipo de resposta no intervalo. Os comportamentos da criança também foram avaliados em nove categorias: desobediência_passi$v a$ (ignorar comandos, pedidos ou perguntas da mãe, não apresentando nenhum tipo de resposta durante o intervalo); inadequação (comportamentos inconvenientes ou inadequados da criança que não apresentam caráter desafiador ou agressivo); insensibilidade (logo após uma resposta de envolvimento positivo da mãe, a criança persiste no comportamento anterior ou ignora o comportamento da mãe); negativismo (comportamentos verbais ou não verbais que indicam raiva, recusa ou desânimo); assertividade (expressão de opiniões ou desejos de forma clara e direta, de maneira não coercitiva); envolvimento positivo (expressão de afeto positivo em relação à mãe como sorrisos, carícias, e demonstração de entusiasmo por meio de elogios ou comentá- rios); obediência (cumprir ou tentar cumprir um comando materno anterior); solicitação (perguntas ou pedidos de ajuda feitos de forma direta ou indireta); brincadeira_independente (a criança brinca sozinha com os brinquedos ou outros objetos e não interage com a mãe). Caso a criança não apresentasse nenhum comportamento em um dado intervalo, a categoria sem resposta era registrada. A codificação das práticas educativas maternas e dos comportamentos infantis foi realizada por dois codificadores independentes (20 horas de treinamento), que desconheciam o grupo ao qual cada díade pertencia. $\mathrm{O}$ índice de fidedignidade entre os codificadores foi calculado em uma amostra de $37 \%$ dos casos (sete vídeos) e atingiu 0,95 (Kappa).

\section{Resultados}

Para avaliar diferenças entre os grupos clínico e não-clínico, foi utilizado o Teste de Mann-Whitney em todas as análises realizadas. Optou-se por um teste não-paramétrico devido ao tamanho da amostra que impediu a distribuição normal dos dados. Foram analisadas as frequências totais de práticas educativas maternas e de comportamentos infantis considerando os quatro episódios interativos avaliados. ${ }^{2}$

A Tabela 3 apresenta frequência média, desvios padrão, mediana, $U$ de Mann-Whitney e nível de significância das práticas educativas, nos grupos clínico e não-clínico nos quatro episódios analisados. Houve diferença significativa apenas na categoria controle assertivo $(U=18 ; p$ $=0,03)$. A análise dos dados revelou que o grupo clínico $(M=15,93 ; D P=7,12)$ apresentou uma média maior do que a do grupo nao clínico $(M=$ $8,91 ; p=4,59)$, ao contrário do que era esperado. Em relação às demais categorias, não houve diferenças significativas.

Análises das práticas maternas e dos comportamentos infantis em cada um dos episódios também foram realizadas, porém, não foram encontradas diferenças significativas entre os grupos clínico e não-clínico. Detalhes sobre os resultados dessas análises podem ser encontrados em AssisSilva (2011). 
Tabela 3

Frequência Média, Desvio Padrão, Mediana, Valor de U e Nível de Significância dos Totais das Práticas Educativas Maternas por Categoria nos Grupos Clínico e Não-Clínico nos Quatro Episódios

\begin{tabular}{|c|c|c|c|c|c|c|}
\hline & \multicolumn{2}{|c|}{ Grupo Clínico $(n=9)$} & \multicolumn{2}{|c|}{ Grupo Não-Clínico $(n=10)$} & \multirow{2}{*}{$U$} & \multirow{2}{*}{$P$} \\
\hline & $M(D P)$ & Md & $M(D P)$ & Md & & \\
\hline Controle ambíguo & $3,96(2,48)$ & 3 & $4,33(4,54)$ & 3,5 & 41 & 0,78 \\
\hline Controle coercitivo & $1,00(1,22)$ & 0 & $0,30(0,67)$ & 0 & 31,5 & 0,28 \\
\hline Insensibilidade & $1,66(2,12)$ & 1 & $0,30(0,67)$ & 0 & 26,5 & 0,13 \\
\hline Intrusividade & $1,44(1,81)$ & 1 & $2,76(1,49)$ & 2,5 & 22,5 & 0,07 \\
\hline Negatividade & $2,11(2,02)$ & 2 & $1,76(1,71)$ & 1 & 42,5 & 0,84 \\
\hline Permissividade & $1,24(2,05)$ & 0 & $0,72(1,39)$ & 0 & 38 & 0,60 \\
\hline Controle assertivo & $15,93(7,12)$ & 15 & $8,91(4,59)$ & 9,7 & 18 & 0,03 \\
\hline Envolvimento positivo & $41,27(8,78)$ & 46 & $39,81(7,96)$ & 41,35 & 37,5 & 0,55 \\
\hline Incentivo & $2,92(5,50)$ & 0 & $1,00(1,94)$ & 0 & 35,5 & 0,45 \\
\hline Sem resposta & $3,18(7,40)$ & 0 & $4,64(5,97)$ & 1,5 & 34 & 0,40 \\
\hline Total de comportamentos maternos & $71,20(19,40)$ & 70,6 & $59,77(13,35)$ & 56 & 25 & 0,11 \\
\hline
\end{tabular}

A Tabela 4 apresenta frequência média, desvio padrão, mediana, $U$ de Mann-Whitney e nível de significância dos comportamentos infantis nos grupos clínico e não-clínico nos quatro episódios analisados. Houve diferenças significativas nas categorias Negativismo, Obediência e também no total de comportamentos infantis. Na categoria Negativismo $(U=19,5 ; p$ $=0,04)$, o grupo clínico $(M=3,42 ; D P=3,79)$ obteve média maior do que o grupo não-clínico
$(M=0,22 ; D P=0,47)$. A mesma tendência foi observada na categoria Obediência $(U=20,5 ; p$ $=0,04)$, em que a média do grupo clínico $(M=$ 13,11; $D P=4,71)$ também foi superior a média do grupo não-clínico $(M=9,19 ; D P=3,96)$. Por fim, no total de comportamentos infantis $(U=$ $17 ; p=0,02)$, as crianças do grupo clínico $(M=$ $5,53 ; D P=15,42)$ novamente obtiveram maior média do que as crianças do grupo não-clínico $(M=42,65 ; D P=13,13)$. Nas demais categorias não houve diferenças significativas.

Tabela 4

Frequência Média, Desvio Padrão, Mediana, Valor de U e Nível de Significância dos Totais dos Comportamentos Infantis por Categoria nos Grupos Clínico e Não-clínico nos Quatro Episódios

\begin{tabular}{lccccccc}
\hline & \multicolumn{2}{c}{ Grupo Clínico $(n=9)$} & \multicolumn{2}{c}{ Grupo Não-Clínico $(n=10)$} & & \\
\cline { 2 - 5 } & $M(D P)$ & $M d$ & $M(D P)$ & $M d$ & & \\
\cline { 2 - 5 } Desobediência Passiva & $1,39(1,69)$ & 0 & $1,02(1,75)$ & 0 & 41 & 0,78 \\
Inadequação & $2,03(2,22)$ & 1,3 & $0,60(1,07)$ & 0 & 26 & 0,13 \\
Insensibilidade & $1,22(1,56)$ & 1 & $1,37(1,94)$ & 0,5 & 42,5 & 0,84 \\
Negativismo & $3,42(3,79)$ & 2 & $0,22(0,47)$ & 0 & 19,5 & 0,04 \\
Assertividade & $9,94(5,58)$ & 10 & $5,83(5,79)$ & 4,2 & 24,5 & 0,09 \\
Envolvimento positivo & $22,13(6,82)$ & 22 & $18,68(8,99)$ & 19,8 & 35 & 0,44 \\
Obediência & $13,11(4,71)$ & 15,5 & $9,19(3,96)$ & 8,65 & 20,5 & 0,04 \\
Solicitação & $4,06(3,41)$ & 2 & $2,04(1,81)$ & 1,5 & 24,5 & 0,09 \\
Brincadeira independente & $1,44(3,28)$ & 0 & $3,70(4,57)$ & 1 & 34 & 0,40 \\
Sem resposta & $8,41(6,95)$ & 8 & $12,97(7,83)$ & 12 & 25,5 & 0,11 \\
\hline Total de comportamentos da criança & $59,53(15,42)$ & 62 & $42,65(13,13)$ & 44,5 & 17 & 0,02 \\
\hline
\end{tabular}




\section{Discussão}

O objetivo deste estudo foi investigar possíveis diferenças entre as práticas educativas de mães de criança com TDAH (grupo clínico) e de mães de crianças sem TDAH (grupo não-clínico) durante a interação mãe-criança. Além disso, foram investigadas possíveis diferenças entre os comportamentos de crianças com TDAH e de crianças sem TDAH.

Foi encontrada apenas uma diferença significativa nas práticas educativas maternas entre os dois grupos. Ao contrário do que era esperado, as mães do grupo clínico apresentaram maior frequência de controle assertivo do que as do grupo não-clínico. Sendo assim, os resultados encontrados não confirmaram as expectativas inicias do estudo quanto às práticas educativas maternas. Em relação ao comportamento infantil, constatou-se que o grupo clínico apresentou mais obediência, negativismo e maior frequência de comportamentos em geral do que o grupo não-clínico. Dessa forma, somente a hipótese que previa maior frequência de negativismo no grupo clínico foi confirmada.

De acordo com Johnston e Jassy, (2007), as características do TDAH aparecem cedo na vida da criança e representam desafios para os pais conseguirem manter práticas educativas consistentes e responsivas. Alguns estudos sinalizaram que pais de crianças com TDAH utilizam mais comandos, repetindo mais vezes o que é solicitado aos filhos do que pais de crianças sem o transtorno (Gau, 2007; Mash \& Johnston, 1982). No presente estudo essa tendência foi constatada, pois as mães do grupo clínico apresentaram mais controle assertivo do que as mães do grupo não-clínico, ou seja, elas utilizaram mais comandos e proibições diretas, na tentativa de direcionar o comportamento dos filhos. Desta forma, a maior frequencia de obediência por parte das crianças com TDAH, poderia ser, pelo menos em parte, explicada por essa tendência das mães de emitirem mais respostas de controle assertivo, que demandam algum tipo de reação ou mudança de comportamento na criança. Por outro lado, a maior dificuldade percebida nos filhos portadores de TDAH em atentar e atender a ordens e pedidos pode fazer com que as mães se enga- gem nesse tipo de prática com maior frequência e de maneira repetitiva. Embora o controle assertivo seja um tipo de estratégia eficaz, que está associada ao desenvolvimento da competência social, é possível que quando utilizada com muita frequência, adquira uma função aversiva e se aproxime do que a literatura caracteriza como intrusividade, por ser uma interferência excessiva no comportamento da criança. Assim, além de explicar a maior frequencia de obediência nas crianças do grupo clínico, a presença excessiva de controle assertivo nas mães, que pode adquir certo caráter estressante e aversivo, também poderia estar associada a maior frequência de negativismo nas crianças desse grupo. A categoria negativismo foi definida como exibir comportamentos verbais ou não verbais que indicam raiva, recusa ou desânimo, ou ainda comportamentos outros que não sejam fazer o que a mãe solicitou, tais como, interromper, ameaçar, provocar, expressar raiva em relação a um pedido ou ordem materna.

Nesse sentido, as reações negativistas das crianças com TDAH poderiam ser compreendidas, pelo menos em parte, como respostas de fuga-esquiva diante do contexto aversivo criado pelos comandos excessivos e repetitivos apresentados pelas mães. Por outro lado, de acordo com Phelan (2005), deficiências no córtex pré-frontal (e suas conexões com os outros centros cerebrais) resultam em um tipo de "governador preguiçoso", e com isso, as atividades do indivíduo com TDAH seriam aleatórias, dispersas e desorganizadas, às vezes muito agressivas e, com frequência, excessivamente emocionais. Assim, o maior número de comportamentos negativistas das crianças do grupo clínico em relação às crianças do grupo não-clínico também pode ser explicado por características do funcionamento neurológico típicas do transtorno, que devem ser levadas em conta.

Quanto às diferenças verificadas no número total de comportamento das crianças dos dois grupos, é importante lembrar que as crianças com diagnóstico de TDAH têm uma série de sintomas relacionados ao déficit de atenção e a hiperatividade/impulsividade. Entre eles, estão: dificuldade para manter a atenção em tarefas lúdicas, ser facilmente distraído por estímulos alheios à tare- 
fa, abandonar sua cadeira em sala de aula ou outras situações nas quais se espera que permaneça sentado, muita agitação e falar em demasia (Rohde \& Halpern, 2004). Além desses sintomas, as crianças com TDAH são mais ativas, inquietas e agitadas ao longo do dia e mesmo durante o sono (Barkley, 2008). Essas características do transtorno, provavelmente, justificam a constatação de que as crianças do grupo clínico emitiram um maior número de comportamentos no somatório de todos os episódios.

Ainda com relação ao achado que revelou que as crianças do grupo clínico emitiram mais comportamentos de obediência do que as crianças do grupo não-clínico, esse resultado, aparentemente controverso cosiderando-se a sintomatologia do TDAH, pode ser explicado por características da observação estruturada que foi utilizada no estudo. Segundo Phelan (2005), crianças com TDAH são capazes de se comportar de forma adequada em situações que tenham uma ou mais das seguintes características: novidade, alto valor de interesse, intimidação ou necessidade de ficar a sós com um adulto. No presente estudo, é possível que a interação diádica analisada tenha apresentado três dessas características: novidade, alto valor de interesse e intimidação. A situação era nova para as crianças, já que elas não conheciam a pesquisadora, as atividades propostas e os brinquedos utilizados. Além disso, algumas crianças se mostraram bastante interessadas em participar da observação, pois os episódios envolviam situações possivelmente reforçadoras como brincar livremente e montar um quebra-cabeça. Além disso, a presença da pesquisadora e da filmadora pode ter funcionado como um fator provocador de intimidação.

Outro dado relevante foi a diferença significativa em relação à presença de transtornos mentais na família e a diferença marginalmente significativa em relação ao abuso de drogas e álcool na família entre os grupos clínico e não-clínico. Algumas pesquisas destacam o fato de que a psicopatologia dos pais, a discórdia marital severa, a classe social baixa e a criminalidade dos pais são fatores ambientais importantes no surgimento ou exacerbação dos sintomas do transtorno (Biederman et al., 1995; Chronis et al., 2007).
Nesse sentido, sugere-se que futuras investigações atentem para a importância desses dados e continuem a investigar esses aspectos.

Outro aspecto que merece registro é que mais crianças no grupo clínico estudavam em escolas particulares do que no grupo não-clínico. A decisão de selecionar crianças de escolas públicas para compor o grupo não-clínico se deu pelo fato de o ambulatório de TDAH atender predominantemente a população de baixa renda. Assim, esperava-se que a totalidade ou a maioria das crianças atendidas no ambulatório frequentasse escolas públicas. De qualquer modo, é importante destacar que as escolas particulares nas quais as crianças do grupo clínico estudavam, podem ser consideradas "escolas de bairro", termo utilizado em Salvador para designar instituições de ensino privadas de pequeno porte que cobram taxas de matrícula e mensalidades de baixos valores (em torno de $\mathrm{R} \$ 150,00$ ), e cuja estrutura, remuneração dos docentes, qualidade de ensino e serviços prestados não se diferencia de forma marcante daquelas encontradas em instituições públicas de ensino. Nesse sentido, é possível pressupor que apesar da diferença observada na freqüência de crianças matriculadas em escolas públicas e privadas nos dois grupos, tais discrepâncias não impliquem diferenças importantes no nível socioeconômico (NSE) das famílias, como indicam os dados da renda e da ocupação dos pais.

Como já foi relatado, as características da sessão de observação podem ter contribuído para o engajamento e interesse das crianças com TDAH nas atividades propostas. É importante considerar essas limitações do método que podem ter, de certo modo, impedido que as diferenças entre os dois grupos emergissem. Além disso, o baixo grau de dificuldade das tarefas pode ter sido um fator determinante para o não surgimento de conflito na interação. Contudo, vale ressaltar que tais episódios foram escolhidos com base em estudos nos quais foram encontradas diferenças significativas entre diferentes grupos analisados (Keown \& Woodward, 2002; Landau et al., 2009; Melnick \& Hishaw, 2000).

Apesar das limitações do presente estudo, os resultados obtidos indicam que o modelo adotado para o presente estudo, que previa que as 
práticas educativas parentais estariam envolvidas no surgimento e manutenção do TDAH, bem como poderiam agravar os sintomas do transtorno, pode não ser o mais adequado. Assim, é preciso considerar e discutir a hipótese de que a os sintomas específicos de TDAH não sofram influência das práticas educativas parentais, como já indicavam alguns dos estudos revisados (Loeber et al., 2000; Whalen \& Henker, 1999).

Caso as práticas educativas parentais, de fato, não estejam relacionadas ao surgimento e exacerbação dos sintomas do TDAH, existe a possibilidade de que os estudos que apontaram para esta relação tenham incorrido em erros de diagnóstico que levaram à seleção equivocada da amostra. Para Phelan (2005), o diagnóstico de TDAH é complexo e repleto de "armadilhas", pois não há um teste específico para determinação do transtorno. Para que o processo de diagnóstico seja seguro e bem delimitado, deve envolver várias etapas, diferentes informantes e instrumentos e entrevistas complementares (Phelan, 2005). Rohde e Halpern (2004) afirmam que o diagnóstico do TDAH também deve envolver avaliações complementares com outros profissionais para a análise das capacidades auditiva e visual, de problemas neurológicos ou neuropiscológicos e de dificuldades psicopedagógicas. Essas informações adicionais facilitam o diagnóstico diferencial do transtorno, bem como auxiliam na detecção da presença ou ausência de comorbidades. Assim, é possível que alguns dos estudos revisados que encontraram diferenças nas práticas educativas maternas tenham selecionado crianças que receberam diagnóstico indevido de TDAH. Por exemplo, uma criança que tem pais permissivos, pode ter um padrão de comportamento impulsivo por não receber limites claros e práticas educativas parentais efetivas e, por isso, poderia ser descrita em inventários e entrevistas como hiperativa ou desatenta pelos pais ou pela professora. Nesse sentido, é possível que alguns estudos refiram-se a crianças portadoras de TDAH, quando na verdade trata-se de outros tipos de problemas emocionais e comportamentais, que fora de um contexto de diagnóstico clínico rigoroso e detalhado, poderiam ter sido confundidos com o TDAH.
No presente estudo, o diagnóstico de TDAH foi realizado de forma muito criteriosa por uma equipe multidisciplinar. Assim, minimizou-se o risco de que crianças com queixas diversificadas fossem consideradas portadoras do transtorno. Esse aspecto metodológico é um ponto forte do estudo e permite que a afirmação da diferença entre os grupos quanto ao controle assertivo por parte das mães e ao negativismo por parte da criança, seja feita com segurança. Esses achados indicam que, embora as práticas educativas maternas não mantenham ou exacerbem os sintomas de TDAH, elas possam exercer alguma função sobre os comportamentos negativistas das crianças, que por sua vez, estão associados ao surgimento de quadros como o TDO e o TC. Nesse sentido, esse achado fornece certo apoio à hipótese de alguns autores (Barkley, 2002; Whalen \& Henker, 1999) de que crianças com TDAH possam desenvolver esse tipo de comorbidade, em parte, pela ação de práticas educativas inadequadas.

Ensuma, embora o presente estudo não tenha confirmado a hipótese inicial quanto às relações entre as práticas educativas maternas e o TDAH, ele apontou para importantes questões relacionadas ao uso do controle assertivo, e também para aspectos metodológicos que precisam ser considerados para que essa problemática seja esclarecida em futuras investigações. Além disso, a ausência de evidências sobre o impacto das práticas educativas parentais no surgimento e manutenção transtorno reforça a hipótese mantida por alguns autores, de que o comportamento parental contribui para o surgimento de comorbidades, mas não tem um papel determinante para o aparecimento ou exacrebação dos sintomas do TDAH. De qualquer modo, fica evidente a necessidade de que novos estudos continuem a examinar as relações entre as práticas educativas e o TDAH, empregando diferentes tipos de delineamentos e instrumentos, envolvendo maior diversidade de informantes, e adotando critérios rigorosos para o diagnóstico do transtorno. Esses avanços são essenciais para que esta importante questão possa ser respondida de forma consistente, e para que, consequentemente, estratégias de intervenção mais eficazes possam ser desenvolvidas. 


\section{Referências}

Alizadeh, H., Applequist, K. F., \& Coolidge, F. L. (2007). Parental self-confidence, parenting styles, and corporal punishment in families of ADHD children in Iran. Child Abuse \& Neglect, 31, 567-572.

Alvarenga, P., \& Piccinini, C. A. (2001). Práticas educativas maternas e problemas de comportamento em pré-escolares. Psicologia: Reflexão e Crítica, 14(3), 449-460.

Alvarenga, P., \& Piccinini, C. A. (2007). O impacto do temperamento infantil, da responsividade e das práticas educativas maternas nos problemas de externalização e na competência social da criança. Psicologia: Reflexão e Crítica, 20(2), 314-323.

Alvarenga, P., \& Piccinini, C. A. (2009). Práticas educativas maternas e indicadores do desenvolvimento do terceiro ano de vida. Psicologia: Reflexão e Crítica, 22(3), 181-190.

American Psychiatric Association. (2000). Diagnostic and statistical manual of mental disorders ( $4^{\text {th }}$ ed.). Washington, DC: Author.

Artigas-Pallarés, J. (2003). Comorbilidad en el trastorno por deficit de atention. Revista de $\mathrm{Neu}$ rología, 36(Supl. 1), S68-S78.

Assis-Silva, L. M. (2011). Práticas educativas de mães de criança com Transtorno de Déficit de Atenção / Hiperatividade (Dissertação de mestrado, Instituto de Psicologia, Universidade Federal da Bahia, Salvador, BA, Brasil).

Bakeman, R., \& Gottman, J. (1999). Observing interaction. Cambridge, UK: University Press.

Barkley, R. A. (2002). Transtorno de Déficit de Atenção/Hiperatividade (TDAH): Guia completo para pais, professores e profissionais de saúde. Porto Alegre, RS: Artmed.

Barkley, R. A. (2008). Etiologias. In R. A. Barkley (Org.), Transtorno de Déficit de Atenção/Hiperatividade: Manual para diagnóstico e tratamento (pp. 31-60). Porto Alegre, RS: Artmed.

Biederman, J., Milberger, S., Faraone, S.V., Kiely, K., Guite, J., \& Mick, E., ...Davis, S. G. (1995). Impact of adversity on functioning and comorbidity in children with Attention-Deficit Hyperactivity Disorder. Journal of the American Academy of Child and Adolescent Psychiatry, 34(11), 1495-1503.
Carlson, E., Jacobvitz, D., \& Sroufe, L. (1995). A developmental investigation of inattentiveness and hyperactivity. Child Development, 66(1), 37-54.

Chronis, A., Lahey, L., Pelham, W., Williams, S., Baumann, B., \& Kipp, H, ...Rathouz, P. J. (2007). Maternal Depression and early positive parenting predict future conduct problems in young children with attention-deficit/hyperactivity disorder. Developmental Psychology, 43, 70-82.

Cypel, S. (2001). A criança com déficit de atenção e hiperatividade: Atualização para pais, professores e profissionais de saúde. São Paulo, SP: Lemos.

DuPaul, G. J., McGoey, K. E., Eckert, T. L., \& VanBrakle, J. (2001). Preschool children with attention-deficit/hyperactivity disorder: Impairments in behavioral, social, and school functioning. Journal of the American Academy of Child and Adolescent Psychiatry, 40(5), 508-515.

Gau, S. S. (2007). Parental and family factors for Attention-Deficit Hyperactivity Disorder in Taiwanese children. Australian and New Zealand Journal of Psychiatry, 41(8), 688-696.

Golfeto, J. H., \& Barbosa, G. A. (2003). Epidemiologia. In L. A. Rohde \& P. Matos (Orgs.), Princípios e práticas em TDAH (pp. 15-33). Porto Alegre, RS: Artmed.

Hoffman, M. L. (1979). Development of moral thought, feeling, and behavior. American Psycologist, 34, 958-966.

Johnston, C., \& Jassy, J. (2007). Attention-deficit/ Hiperactivity Disorder and Oppositional/conduct Problems: Links to parent-child interactions. Journal of the Canadian Academy of Child and Adolescent Psychiatry, 16(2), 74-79.

Johnston, C., \& Mash, E. (2001). Families of children with attention-deficit/hyperactivity disorder: Review and recommendations. Clinical Child and Faily Psychology Review, 4(3), 183-207.

Keown, L. J., \& Woodward, L. J. (2002). Early parent-child relations and family functioning of preschool boys with pervasive hiperactivity. Journal of Abnormal Child Psychology, 30(6), 541-553.

Kunrath, L. H., Wagner, A., \& Jou, G. Y. (2006). A educação dos filhos com Transtorno de Déficit de Atenção de Hiperatividade: O que fazer? Psicologia em Revista, 12(20), 235-250. 
Lambert, N. M., Sandoval, J., \& Sassone, D. (1978). Prevalence of hyperactivity in elementary school children as a function of system definers. American Journal of Orthopsychiatry, 48, 446-463.

Landau, R., Amiel-Laviad, R., Berger, A., Atzaba-Poria, N., \& Auerbach, J. (2009). Parenting of 7-month old infants at familial risk for ADHA during infant's free play, with restrictions on interaction. Infant Behavior \& Development, 32, 173-182.

Loeber, R., Green, S., Lahey, B., Frick, P., \& McBurnett, K. (2000). Findings on disruptive behavior disorders from the first decade of the Developmental Trends Studies. Clinical Child and Family Psychology Review, 3, 37-60.

Mash, E. J., \& Johnston, C. (1982). A comparison of the mother-child interactions of younger and older hyperactive and normal children. Child Development, 53, 1371-1381.

Mattos, P., Serra-Pinheiro, M. A., Rohde, L. A., \& Pinto, D. (2006). Apresentação de uma versão em português para uso no Brasil do instrumento MTA-SNAP-IV de avaliação de sintomas de transtorno do déficit de atenção/hiperatividade e sintomas de transtorno desafiador e de oposição. Revista de Psiquiatria do Rio Grande do Sul, 28(3), 290-297.

Melnick, S. M., \& Hinshaw, S. (2000). Emotion Regulation and Parenting in $\mathrm{AD} / \mathrm{HD}$ and comparison Boys: Linkages with social behaviors and peer preference. Journal of Abnormal Child Psychology, 28(1), 73-86.

Pacheco, J. T. B., Silveira, L. M. O. B., \& Schneider, A. M. A. (2008). Estilos e práticas educativas parentais: Análise da relação desses construtos sob a perspectiva dos adolescentes. Psico, 39(1), 66-73.

Patterson, G. R., DeGarmo, D. S., \& Knutson, N. (2000). Hyperactive and antisocial behaviors: Comorbid or two points in the same process? Development and Psichology, 12, 91-106.

Patterson, G. R., Reid, J., \& Dishion, T. (1992). Antisocial Boys. Eugene, OR: Castalia.
Phelan, T. W. (2005). TDA/TDAH: Transtorno de Déficit de Atenção e Hiperatividade: Sintomas, diagnósticos e tratamento: Crianças e Adultos. São Paulo, SP: M. Books do Brasil.

Poeta, L. S., \& Rosa, F., Neto. (2004). Estudos epidemiológicos dos sintomas de Transtorno do Déficit de Atenção/Hiperatividade e Transtornos de Comportamento em escolares da rede pública de Florianópolis usando a EDAH. Revista Brasileira de Psiquiatria, 26(3), 150-5.

Rohde, L. A., Barbosa, G., Tramontina, S., \& Polanczyk, G. (2000). Transtornos de déficit de atenção/hiperatividade. Revista Brasileira de Psiquiatria, 22(Supl. 2), 7-11.

Rohde, L. A., \& Halpern, R. (2004). Transtorno de déficit de atenção/hiperatividade: Atualização. Jornal de Pediatria, 80(Supl. 2), 61-70.

Roman, T., Schmitz, M., Polanczyk, G. V., \& Hutz, M. (2003). Etitologia. In L. A. Rohde \& P. Mattos, Principios e Práticas em TDAH: Transtorno de Déficit de Atenção / Hiperatividade (pp. 3552). Porto Alegre, RS: Artmed.

Sell-Salazar, F. (2003). Sindrome de hiperactividad y deficit de atention. Neurología, 37(4), 353-358.

Swanson, J. M. (1992). School-based assessment and interventions for ADD students. Irvine, CA: K.C.

Tannock, R. (1998). Attention-deficit/hyperactivity disorder: Advances in cognitive, neurobiological and genetic research. Journal of Child Psychology and Psychiatry, 39, 65-99.

Whalen, C. K., \& Henker, B. (1999). The child with attention deficit hyperactivity disorder in family contexts. In H. C. Quay \& A. E. Hogan (Eds.), Handbook of disruptive behavior disorders (pp. 139-155). New York: Plenum. 
Apêndice

Atividade de Cópia

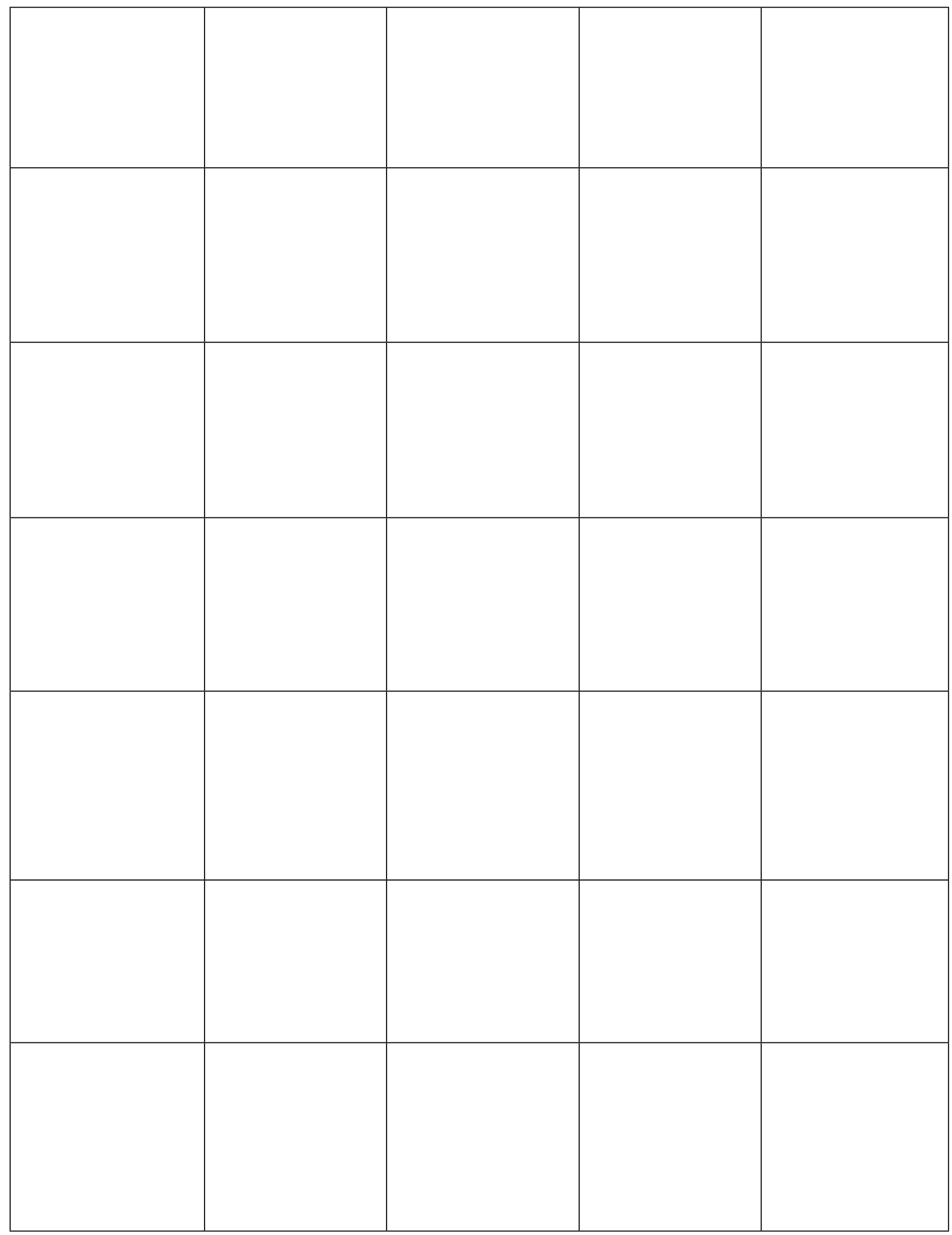

